\title{
The application of holography laser for the assessment of bone-implant fixation conditions
}

\author{
J. A. Simões' \& M. Vaz? \\ 'Departamento de Engenharia Mecânica, Universidade de \\ Aveiro, Portugal \\ 'Departamento de Engenharia Mecânica e Gestão Industrial. \\ Universidade do Porto, Portugal
}

\begin{abstract}
Within this paper, the study of different bone-implant interface conditions (bonded versus debonded implants) using Electronic Speckle Pattern Interferometry and the Finite Element Method is presented and discussed. For the experimental study, bovine cancellous bone was used with a metal tapered rod simulating the femoral component, which was centrally inserted in cancellous bone. The simplified femur-prosthesis construction was sectioned to expose the bone-implant interface and the displacement/stress distributions obtained. A qualitative good correlation between the numerical and experimental results was obtained. The results evidenced that the load is transferred in the immediate vicinity of the implant to the surrounding cancellous bone in different ways, depending on the interface bonding characteristics.
\end{abstract}

\section{Introduction}

In these last years, significant incremental application of experimental investigation methods has been used to study biomechanical structures. The necessity to quantify loads, stresses and strains in bone structures has pushed the development of new and more powerful techniques for experimental analysis. These new techniques, widely applied in the study of other engineering problems, have been adapted to satisfy the inherent constraints of anatomical structures. The development of numerical techniques, namely those based on the finite element method, has allowed the simulation of very complex anatomical structures with great reliability. Due to the complex nature of biomechanical problems, it is a wise criterion that experimental output data can be validated numerically, or vice-versa. 
The word holography was proposed by Dennis Gabor to name a process that allows the recording of all information available in a wave front, its phase and amplitude distributions [1]. With this technique, it is possible the three dimensional reconstruction of the recorded wave front with all of its characteristics. Holographic interferometry techniques rely on the interference between wave fronts obtained from holographic recordings of objects in different loading conditions. It is therefore possible to accurately assess the three dimensional displacement distributions in the micron range. The global nature of the measurement, which is obtained with no contact or special transducer, recommends this technique for a wide range of applications in different fields such as experimental mechanics [2], defect detection [3] and biomechanics [e. g. 4], among others.

In the classical set-up, holograms were recorded on high resolution photographic emulsions that were chemically developed. This procedure implies a slow and cumbersome process that contributed to the slow acceptance of holographic interferometry in the scientific community, in spite of their potential. The introduction of the electronic video recording of holograms in the beginning of the seventies makes this technique working in real time at high sampling rates (25/30 holograms/s European/US video standard) [5]. This set-up is commonly known by ESPI that stands for Electronic Speckle Pattern Interferometry.

Holographic interferometry has become more user friendly and its diffusion has increased significantly. Finally, the digital video systems, directly connected to personal computers bus, allow the use of image processing routines to improve and speed up the data analysis. Nowadays, holographic interferometry techniques are frequently used in several different scientific domains were displacement fields have to be assessed with very high resolution. Figure 1 illustrates schematically a typical ESPI set-up.

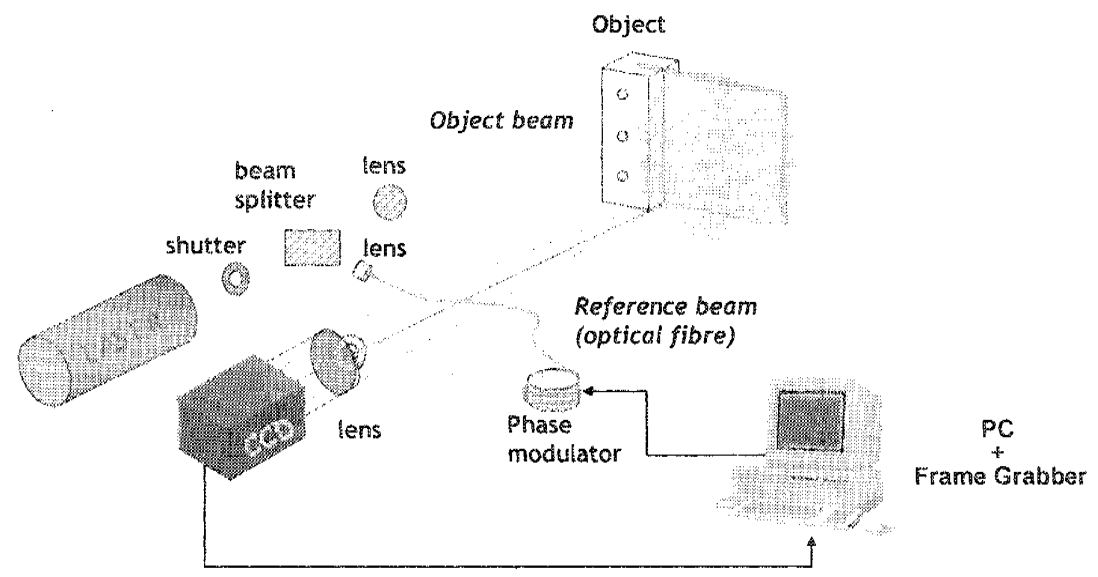

Figure 1 - ESPI set-up with image processing capabilities. 
Most conventional methods of measuring surface strain and displacement are based on photoelastic, strain gauges, dial gauges and other mechanical and electrical sensing devices. These measuring methods can be highly sensitive and accurate, but give information in limited regions of the structure. To obtain the strain or displacement distribution of bigger areas it is necessary to apply full field techniques, such as Moiré and Speckle. Moiré techniques show in -plane displacements distributions in a continuous manner over the entire surface but its sensitivity depends on the grid pitch. When holographic interferometry techniques are used to perform measurements, the set-up can be adjusted to orient the sensitivity vector according to a prescribed direction. In-plane and outof-plane measurements can be easily obtained on diffuse surfaces with none or very small surface preparation. Sometimes the object needs to be gently painted to improve its reflectivity. Combinations of set-ups allow the simultaneous acquisition of recordings that can be processed to obtain the three dimensional displacement maps. These results can be inputted in numerical routines to obtain its spatial differentiation that correspond to strain fields.

Speckle techniques can also be used to measure in-plane displacement distributions. It is a non-contact field technique that makes use of the speckle patterns resulting from a coherent illumination of a rough surface. This technique has been widely used for accurate measurements of the deformation patterns of structures when exposed to mechanical or other type of stresses. One of its advantages relies on its high sensitivity; displacements of the order of the wave length can de assessed. The results within this method are obtained in the form of fringe patterns, each fringe corresponding to points having the same displacement in the direction of the sensitivity vector.

The study of porous structures, like trabecular cancellous bone, using optical methods has been addressed [6-10]. Bay [6] used texture correlation to measure the displacement and strain patterns within samples of trabecular bone. This technique is a modification of digital image correlation, a method for analysis of deformation in objects marked with random surface speckle. Due to the cancellous bone structure characteristics, and instead of surface speckle, the trabecular pattern itself is used as a basis for correlation. An experimental set-up using speckle interferometry was developed by Simões et al. [7] to measure the displacement patterns of a foam material, replicating cancellous bone. The results of this work show that foam-implant interface conditions are relevant on the definition of the displacement pattern; suggesting that the load applied to the implant is transferred to the surrounding foam media in different ways.

\section{Materials and methods}

A fresh bovine femur was used in this study. Part of the femur was sectioned in the frontal plane and the cancellous bone of the proximal femur was fixed with an epoxy resin to the test rig. The prosthesis was idealised as a metal tapered rod inserted in cancellous bone. The model was sectioned longitudinally to expose 
the bone-implant interface. Figure 2 shows the bone specimen and the implant used. The test rig used by Simões et al. [8-10] was optimised to correctly measure the applied forces and to avoid boundary effects in the displacement measurements. The rig was designed to enclose the bone, and to have sufficient rigidity to avoid rigid body displacements due to the load applied to the implant. For the bonded situation, the implant was glued with a very thin layer to the bone. It was observed that the glue did not penetrate deeply the cancellous bone structure and therefore did not reinforce it at the contact area. A constant $0,1 \mathrm{~mm}$ displacement was applied to the implant and fringe patterns recorded [8-11].

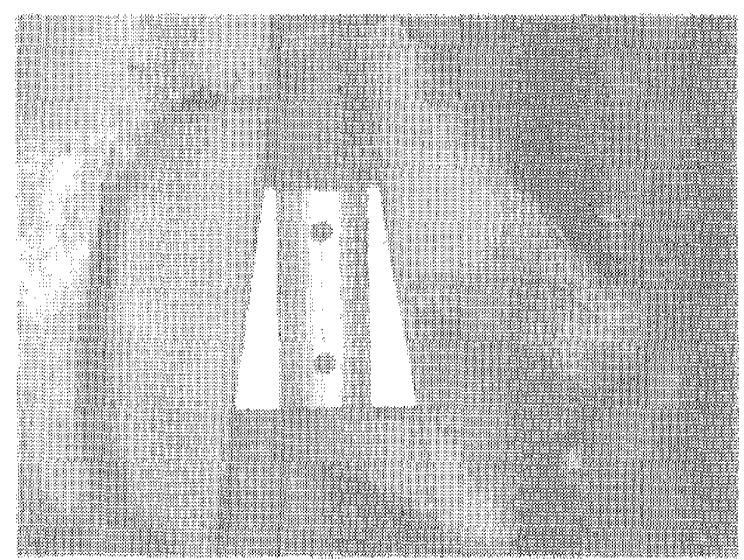

Figure 2 - Bone and implant model studied.

Within the finite element simulation, the cancellous bone structure and implant were modelled using SolidWorks(B) 2001 computer aided design software and the analysis performed with Cosmos/WORKS 6.0 and ABAQUS. The finite element model was composed of 19095 tetrahedral solid elements and 28190 nodes. Figure 3 shows the finite element mesh and the CAD model. Since high stress concentrations occur at the edges of the implant, the mesh was sufficiently refined at these regions.

An elastic modulus of $0.4 \mathrm{GPa}$ and $20 \mathrm{GPa}$ was assigned respectively to the cancellous and cortical bone structure. For the implant, an elastic modulus of 210 $\mathrm{GPa}$ was considered. A Poisson's ratio of 0.3 was used for all materials involved in the simulation problem. For the debonded interface, and since the coefficient of friction between the implant and the surrounding cancellous bone was unknown, the numerical simulations were performed for $\mu=0.0, \mu=0.1$ and $\mu=0.3$.

For the experimental study, a set-up with four directions of illumination was used to measure the in-plane displacement field at the surrounding bone due to the axial movement of the implant (figure 4). In this set-up a liquid crystal retarder (LCR) was used to shift the illumination from one direction to the other. 

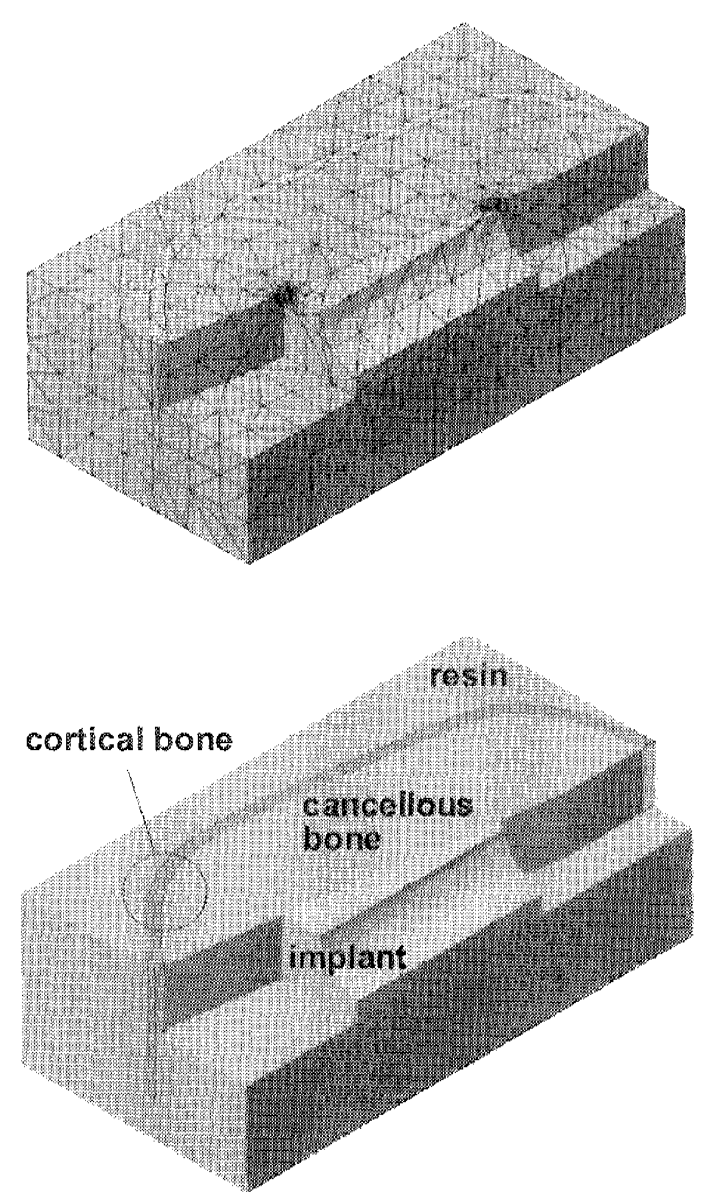

Figure 3 - Finite element mesh and CAD model.

The set-up is a duplication of the one proposed by Ennos [11] to assess in-plane displacements. In this case, using a special routine of image processing, it was possible to record two different references and obtain the displacement distributions in two different directions (axial and transverse). Within the holograms fringe patterns, each fringe represents points of an equal displacement in the sensitivity vector direction. An image processing system was used to calculate the phase.

\section{Results and discussion}

The displacement fields in the region of the bone surrounding the implants were assessed in the axial and transverse directions. For both cases, a constant displacement was applied to the implant and it was observed that qualitatively the fringe pattern did not depend on the magnitude of the displacement. However, if 
higher implant displacements were imposed, higher number of fringes were generated, being difficult the read and interpret them. The experimental and numerical fringe patterns for the bonded and debonded implants, in the axial and transverse directions, are shown in figure 5. The first two images correspond to the experimental and numerical displacement patterns within the axial direction; the other two correspond to the same displacements in the transverse direction. The axial direction corresponds to the direction of the prescribed displacement.

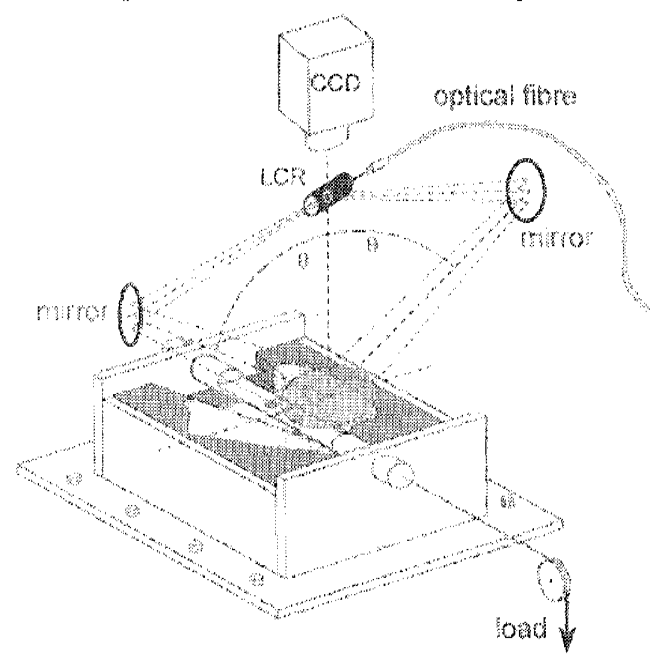

Figure 4 - LSPI set-up for measurement of in-plane displacements.
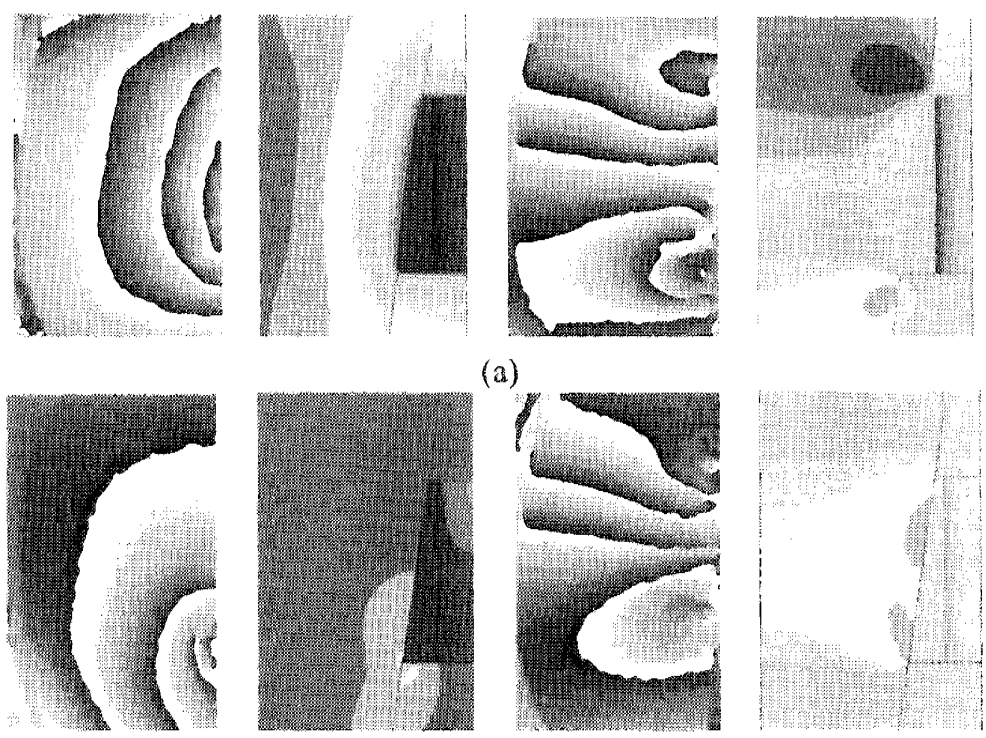

(b)

Figure 6-Experimental and numerical fringe pattems in the axial and transverse directions: a) bonded implant; b) debonded implant. 
Very few studies have been done to assess the stress-strain distribution due to implants inserted in cancellous bone. Preliminary studies performed by Simões et al. [7-10] showed that ESPI is a suitable experimental technique to assess displacements within porous materials and therefore applicable to the examination of interface bone-implant failure mechanisms. Within this study, it was observed that for the bonded situation, those points adjacent to implant and bone move together since they are bonded. Regions of pronounced displacement concentrations were observed at the implant's edges. For the axial direction, it was seen that the iso-displacement fringe pattern for the bonded interface was uniformly distributed, where for the debonded one, a localised gradient of displacements was observed at the edge of the implant were the displacement was prescribed. The debonded implant generated much higher magnitude transverse displacements and provoked a high displacement gradient at the distant edge relatively to the loading one. For the bonded implant this observation was not so pronounced. The FEM analysis was performed to compare the obtained displacement field with the one obtained using ESPI, and a good qualitatively agreement was obtained for both methods used. This gave the necessary confidence to make the discussion of the work based on the numerical stresses.

Figure 6 identifies the normal interface stress distributions for the bonded and debonded implant respectively. Simulations realised for the debonded implant using different coefficients of friction showed very similar results. The shear stress showed a similar distribution for both types of interfaces simulated. The mean shear stresses for the bonded implant is $0.01 \mathrm{MPa}$ and is mainly due to peak shear stresses at the edges of the implant. Between these edges, there are practically no shear stresses for either bonded or debonded interfaces.

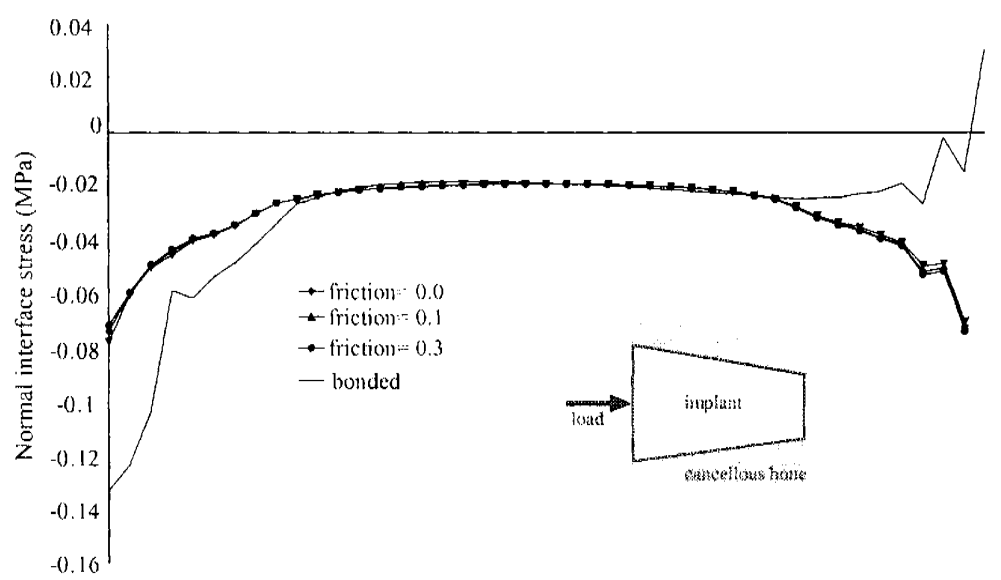

Figure 6 - Normal interface stresses (bonded implant) [10].

The main conclusion of the numerical study is that the load applied is mainly transferred to the surrounding cancellous bone at the regions adjacent to the edges of the implant. In fact, it can be observed that between the edges of the implant, 


\section{Simulations in Biomedicine $V$}

the normal stresses are comparatively lower. As said before, no significant shear stresses were observed between the implant's edges. The discussion on the load transfer mechanism is somewhat difficult if only based on the normal and shear stress magnitudes. In fact, it must be said that the stress values at regions of high stress concentrations must be carefully analysed due to their singularity characteristics.

Due to the nature of the interface, the debonded implant transfers the load mainly through the normal stresses. The mean shear stresses are very low, of the order of $-0.01 \mathrm{MPa}$. If the mean shear stresses are considered, it can be seen that for the bonded interface they are $96 \%$ higher. The mean normal interface stresses for, both bonded and debonded implants, are similar, of the order of $0.04 \mathrm{MPa}$, which means that the stresses are much higher between the edges of the debonded interface. The differences observed between the bonded and debonded implant is mainly due to the mechanism related on how the nodes belonging to the cancellous bone surface move when they are fixed to the implant, or when not. Bone and implant interface nodes move together in a direction provoked by the displacement imposed to the bonded implant; the debonded bone interface nodes also move due to a relatively more pronounced wedging effect of the implant, allowing the implant to subside inside the bone, but, in this case, in an opposite transverse trajectory relatively to the bonded nodes. This effect was not so markedly observed for the implant fixed to the cancellous bone.

\section{Conclusions}

In the immediate vicinity of the implant, the fringe patterns were similar to those predicted by finite element models, for both bonded and debonded conditions and for the axial and transverse displacements. The debonded implant generated much higher displacements and the nodes adjacent to the implant move in the transverse direction mainly due to the wedging of the implant, allowing the implant to subside inside the bone structure. This phenomenon was not so markedly observed for the bonded implant. Relatively to the load transfer mechanism, it was observed that the load is transferred mainly at the regions of high stress concentrations; this is, at the edges of the implant.

\section{Acknowledgments}

The authors gratefully acknowledge the Fundação para a Ciência e a Tecnologia do Ministério da Ciência e do Ensino Superior for funding through projects POCTI/EME/36345/1999 and POCTI/EME/38367/2001.

\section{References}

[1] Gabor; D., 1948, "A New Microscopic Principle", Nature, 161 (4098), pp. 777. 
[2] Powell, R. L., Stetson, K. A., 1965, "Interferometric Vibration Analysis by Wavefront Reconstruction"; J. of Optical Soc. of America; 55 (12), pp. 1593.

[3] Waters, J. P., 1974, "Holographic Non Destructive Testing", Erf, K. R. (Ed); Academic Press, Inc., New York, pp. 5.

[4] Svensson, L. B. M., Wos, H., 1987, "A Metrological Study of Holographic Measurements of Vibration Applied to the Back of the Human Body", L.I.A., 60, ICALEO, pp. 103.

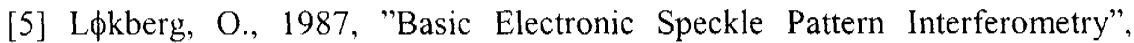
Applied Optics and Optical Engineering, Vol. X, Academic Press, pp. 455.

[6] Bay, K. B., 1995, "Texture correlation: a method for the measurement of detailed strain distributions within trabecular bone", J. of Orthopaedic Res., 13 , pp. 258.

[7] Simões, J. A., Vaz, M. P., Chousal, J. A., Taylor, M., Blatcher, S., 1997, "Speckle interferometry to measure the strain distribution within porous materials", International Conference on Advanced Technology in Experimental Mechanics, ATEM'97, 25-26 July, The Japan Society of Mechanical Engineers and Materials and Mechanics Division, JSME-MMD, pp. 423, Wakayama, Japan.

[8] Simões, J. A., Monteiro, J., Vaz, M. A., Taylor, M., Blatcher, S., 1998, "Study with speckle interferometry of bone-implant interface conditions", Proc: of the 1/th International Conference on Experimental Mechanics, 2428 August, Oxford, United Kingdom, Experimental Mechanics, I. M. Allison, ed., A.A. Balkema, Rotterdam, pp. 851.

[9] Simões, J. A., Monteiro, J., Chousal, J. A., Vaz, M. A., Taylor, M., 2000, "Strain patterns adjacent to bonded and debonded bone-implant interfaces: A holographic study of an idealised proximal femur", Proc. of the $12^{\text {th }}$ Conference of the European Society of Biomechanics, 28-30 August, P. J. Prendergast, T. C. Lee and A. J. Carr, eds., Dublin, pp. 201.

[10] Simões, J. A., Morais, A., Monteiro, J., Vaz, M. A., Taylor, M., 2001, "Numerical and experimental investigation of bone-implant interfaces", $J$. Biomechanics, Vol. 34, Suppl. 1, S85-S86, Proc. of the BioMechanica IV, 23-25 September, K. Ito, M. Morlok, E. Schneider e M. Wimmer (Guest Editors), Davos, Switzerland.

[11] Ennos, A. E., 1968, "Measurement of in-plane surface strain by hologram interferometry", J. of Scientific Instruments (J. of Physics E), Series 2, 1, pp. 731. 
